\title{
Specific stacking angles of bilayer graphene grown on atomic-flat and -stepped $\mathrm{Cu}$ surfaces
}

\author{
Hyeyeon Cho ${ }^{1,3}$, Yohwan Park ${ }^{1,3}$, Soyoung Kim ${ }^{1}$, Taemin Ahn ${ }^{2}$, Tae-Hwan Kim $\mathbb{D}^{2}$ and Hee Cheul Choi $\mathbb{D}^{1 凶}$
}

\begin{abstract}
Bilayer graphene (BLG) exhibits unique properties depending on a stacking angle between the two layers of graphene. Although it has been known that BLGs having stacking angles of $0^{\circ}$ and $30^{\circ}$ can be obtained by chemical vapor deposition (CVD), not much is known yet about the effect of copper $(\mathrm{Cu})$ surface on the decision of stacking angle, through which further fine control of the stacking angle could be possible. Here, we report that the crystal plane of Cu catalyst plays a critical role in the selection of the stacking angle of BLG, and provide experimental and computational evidence that an atomic-flat Cu (111) surface generates BLGs having $0^{\circ}$ and $30^{\circ}$ of stacking angle, while atomic-stepped $\mathrm{Cu}(311)$ and $\mathrm{Cu}(110)$ surfaces mainly produce small stacking angle BLGs with $3-5^{\circ}$ of stacking angle as a major product by CVD.
\end{abstract}

npj 2D Materials and Applications (2020)4:35; https://doi.org/10.1038/s41699-020-00169-x

\section{INTRODUCTION}

Bilayer graphene (BLG), stacked two layers of single-layer graphene (SLG), has attracted a great deal of attention in recent years for valuable fundamental studies in physical sciences and for future device applications ${ }^{1-5}$. Especially, the stacking angle $\left(\theta^{\circ}\right)$ control of BLG arises as an important research subject, as it shows unique physical properties depending on $\theta^{\circ 6,7}$. For example, superconductivity is observed from BLG having $1.1^{\circ}$-twisted stacking angle ${ }^{8-10}, \theta^{\circ}$-tunable photoluminescence ${ }^{11}$, and $\theta^{\circ}$-dependent van Hove singularity ${ }^{12,13}$. For the successful and systematic studies of the correlation between the stacking angle of BLG and the above-mentioned properties, it is highly required to control the stacking angle of BLG, ideally upon its synthesis.

Chemical vapor deposition (CVD) is one of the most popular synthesis method for the growth of SLG using metal catalysts ${ }^{14-16}$. However, in the case of BLG, only the most energetically stable AB-stacked (also called Bernal-stacked) BLG has been mainly obtained by $\mathrm{CVD}^{17-19}$. Thus, the synthesis of BLGs with controlled stacking angles by CVD method has been limited. Many papers have shown the exclusive formation of BLGs having $0^{\circ}-$, and $30^{\circ}$ twisted stacking angles on $\mathrm{Cu}$ (111) surface by CVD, which gives an important sign for the possibility of stacking angle control ${ }^{20,21}$.

Various transition metals like $\mathrm{Pt}, \mathrm{Ni}, \mathrm{Au}$, and $\mathrm{Cu}$ have been examined as a catalyst for synthesizing BLG by CVD method ${ }^{22-25}$. Among them, $\mathrm{Cu}$ has been most widely used because it follows a surface-mediated growth mechanism that allows atomic-scale control on the BLG synthesis ${ }^{26-28}$. Since BLGs are also supposed to be synthesized directly on $\mathrm{Cu}$ by surface reaction, the characteristic of the Cu surface is supposed to affect the BLG growth and its stacking angle, as BLG is known to grow on $\mathrm{Cu}$ surface through underlayer growth mechanism ${ }^{29,30}$. However, no clear experimental evidence supporting the stacking angle dependence on the Cu surface is available yet.

Herein, we report an experimental demonstration of the direct relationship between the structure of $\mathrm{Cu}$ surface and the stacking angle of BLG. It is well-known that $\mathrm{Cu}(111)$ that has an atomic-flat surface induces $A B$-stacking and $30^{\circ}$-twisted stacking in $B L G^{20}$. During our own reproducibility tests, we have found that BLGs having other twisted stacking angles (especially preferred stacking angle below $5^{\circ}$ ) are also formed on $\mathrm{Cu}$ foils that contain $\mathrm{Cu}(311)$ and (110) surfaces. That is, such BLGs are formed when the Cu has other crystal planes than the (111) crystal plane. These findings motivate us to investigate the correlation between the structure of $\mathrm{Cu}$ surface and the stacking angle of BLG. To reveal the correlation, we synthesized BLGs on both traditional $\mathrm{Cu}$ (111) (atomic-flat surface) and $\mathrm{Cu}(311) /(110)$ (atomic-stepped surface). As a result, small-angle twisted BLGs of which stacking angles ranging from $3^{\circ}$ to $5^{\circ}$ were grown mostly on atomic-stepped $\mathrm{Cu}$ surfaces such as $\mathrm{Cu}(110)$ and $\mathrm{Cu}(311)$, while AB-stacked and $30^{\circ}$ twisted BLGs were mainly obtained on atomic-flat $\mathrm{Cu}$ (111) surface as reported. Our results reveal that the surface structure of $\mathrm{Cu}$ indeed affects determining the stacking angle of BLG, which may allow fine control of the stacking angle of BLG.

\section{RESULTS AND DISCUSSION}

Reconstruction of BLG growth on Cu (111) surface

BLGs are known to be formed by a sequential growth of the upper layer of graphene (first) and the lower layer graphene (second) on $\mathrm{Cu}$ surface by $\mathrm{CVD}^{31,32}$. In this case, both graphene layers share the same nucleation site of $\mathrm{Cu}$ for their growths. Both theoretical and experimental studies have shown that $A B$-stacked BLGs and $30^{\circ}$-twisted BLGs are mainly formed at $\mathrm{Cu}$ (111) surface due to their high thermodynamic stability based on the favorable lattice interaction between $\mathrm{Cu}(111)$ and $\mathrm{BLGs}^{33,34}$. To verify this and further examine such an effect of Cu crystal plane on the stacking angle of BLGs, we attempted to reproduce the growth of BLGs on Cu (111) by CVD.

$\mathrm{Cu}$ containing (111) crystal planes are obtained by annealing commercial $\mathrm{Cu}$ foils at $1000^{\circ} \mathrm{C}$ under $\mathrm{Ar}$ and $\mathrm{H}_{2}$ gas environment. Details about the reaction condition are described in the "Methods" section. The crystal plane of the annealed $\mathrm{Cu}$ is identified by X-ray diffraction (XRD) analysis. Before annealing, commercial $\mathrm{Cu}$ foils have various crystal planes such as (111), (200), (110), and (311) with low crystallinity (Supplementary Fig. 1). After annealing, (111) crystal plane that has an atomic-flat

${ }^{1}$ Department of Chemistry, Pohang University of Science and Technology (POSTECH), Pohang 37673, Republic of Korea. ${ }^{2}$ Department of Physics, Pohang University of Science and Technology (POSTECH), Pohang 37673, South Korea. ${ }^{3}$ These authors contributed equally: Hyeyeon Cho, Yohwan Park. ${ }^{凶}$ email: choihc@postech.edu 
(a)

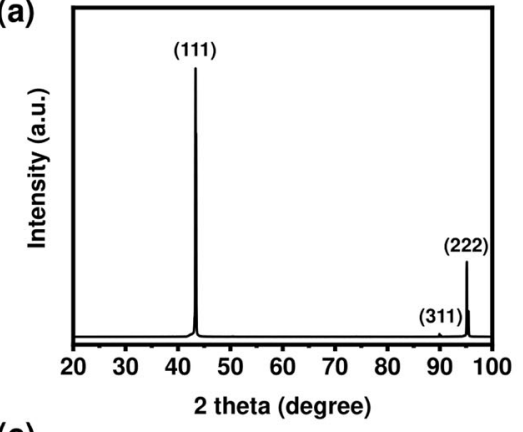

(c)

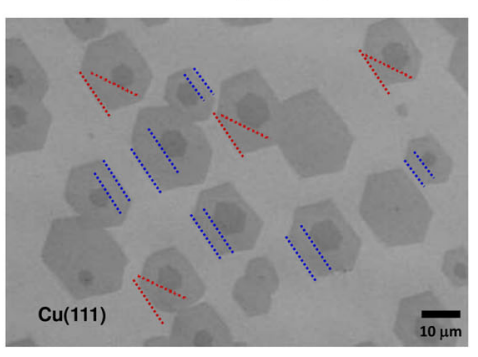

(b)

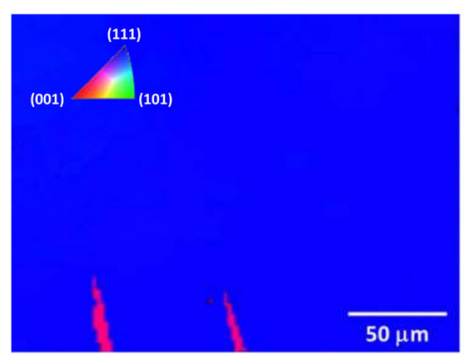

(d)

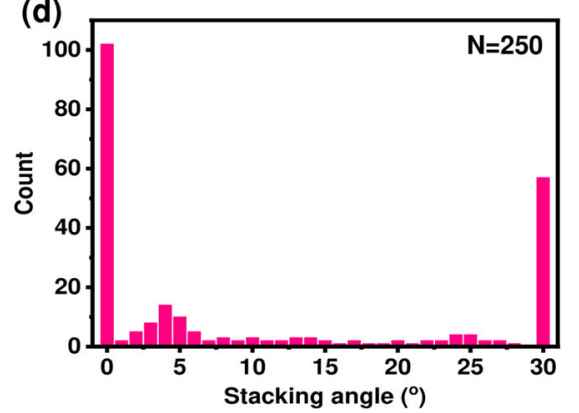

Fig. 1 Characterization of $\mathrm{Cu}$ (111) and grown BLG. a XRD pattern of $\mathrm{Cu}$ (111). b EBSD mapping image of Cu (111). The inset is the color coding orientation triangle. The blue color region is (111) plane, and the pink color region is (311) plane. c SEM image of BLG/Cu (111). BLGs grown on $\mathrm{Cu}(111)$ have $\mathrm{AB}$-stacking or $30^{\circ}$-twisted stacking order. The stacking angle is determined by dashed lines. AB-stacked BLG (blue dashed line) has $0^{\circ}$ of stacking angle. $30^{\circ}$-twisted BLG (red dashed lines) has $30^{\circ}$ of stacking angle. $\mathbf{d}$ Stacking angle distribution of BLG grown on $\mathrm{Cu}(111)$.

structure is still the major plane as shown in Fig. 1a. More importantly, (311) crystal plane that has an atomic-stepped structure also appears on $\mathrm{Cu}$ surface at $2 \theta$ of nearly $90^{\circ 35}$ (Supplementary Fig. 2). An electron backscatter diffraction (EBSD) mapping image shows that Cu surface mostly has a blue color that corresponds to the (111) crystal plane, but partially has (311) crystal plane that is marked in pink color (Fig. 1b). It indicates that the annealing of $\mathrm{Cu}$ foil does not produce a perfect surface with (111) crystal plane, but also with other crystal planes. When BLG growth is conducted on $\mathrm{Cu}$ (111), we confirmed that AB-stacked BLGs and $30^{\circ}$-twisted BLGs are the major products that are the same results as previous reports ${ }^{20,21}$.

\section{Characterization of BLG growth on $\mathrm{Cu}$ (111) surface}

The shape and stacking angle of BLGs grown on $\mathrm{Cu}$ (111) can be confirmed by scanning electron microscopy (SEM). Figure 1c shows that most of BLGs have $\mathrm{AB}$-stacking order or $30^{\circ}$-twisted stacking order when they grow on $\mathrm{Cu}(111)$. The rotated angles of $A B$-stacked BLGs are indicated with blue dashed lines and $30^{\circ}$ twisted BLGs with red dashed lines. The first and second layer of $A B$-stacked BLG are parallel to each other, which implies that the stacking angle is $0^{\circ}$ (Supplementary Fig. 3a). The selected area electron diffraction (SAED) pattern corresponding to AB-stacked BLG is shown in the inset of Supplementary Fig. 4b. The spot intensity profile of SAED pattern shows that the $(1210)$ intensity is 1.6 times stronger than $(0 \overline{1} 10)$ intensity, which is different from SLG (Supplementary Fig. $4 a$ ). In the case of $30^{\circ}$-twisted BLG, the first and second layers are angled at $30^{\circ}$, and the corresponding SAED pattern shows two hexagonal patterns that are twisted by $30^{\circ}$ (Supplementary Figs. 3b and 4c).

Interestingly, small numbers of BLGs that are not $A B$-stacked $\mathrm{BLGs}$ or $30^{\circ}$-twisted BLGs, but BLGs with small stacking angles are also observed. Figure $1 \mathrm{~d}$ shows the stacking angle distribution of BLGs grown on $\mathrm{Cu}$ (111), and we examined $250 \mathrm{BLGs}$ for the statistical analysis. As a result, $A B$-stacked BLG and $30^{\circ}$-twisted BLG are $40.8 \%$ and $27.8 \%$, respectively, and the rest of BLGs have a twisted stacking angle. Interestingly, most of the twisted BLGs have small angles, especially below $5^{\circ}$. From these results, $\mathrm{Cu}(311)$ plane, which is known as atomic-stepped surface, is believed to be the origin of small-angle twisted stacking of BLG, and led us to revisit the effect of the crystal plane on BLG growth and its stacking angle determination ${ }^{36}$.

Synthesis of BLG on Cu (311)/(110) surface

To further verify the effect of atomic-stepped surface on the formation of small-angle twisted BLGs, we prepared $\mathrm{Cu}$ having atomic-stepped surface by annealing commercial Cu foils at $800^{\circ} \mathrm{C}$ for $2 \mathrm{~h}$. When polycrystalline $\mathrm{Cu}$ is thermally annealed, $\mathrm{Cu}$ atoms migrate by thermal energy in a way to decrease the surface energy of Cu surface, and a Cu (311)/(110) surface is obtained as thermodynamic products ${ }^{37}$. As shown in Fig. 2a, (110) and (311) crystal planes appear with high crystallinity than the original $\mathrm{Cu}$ foil in XRD pattern. To characterize the $\mathrm{Cu}$ (311) and (110) surface, we performed scanning tunneling microscope (STM) studies and measured the interterrace distances (Supplementary Fig. 5a, b). Their average interterrace distances were confirmed to be $2.6 \pm$ $0.03 \AA$ and $1.1 \pm 0.08 \AA$ from the height profiles of terraces according to the interplanar spacing formula ${ }^{38}$, indicating that the terraces are (110) and (311) crystal planes of fcc $\mathrm{Cu}$, respectively (Supplementary Fig. 5c, d). According to the wellestablished theories and previous studies, the atomic arrangements of these planes have the atomic-stepped surface, while the (111) has the atomic-flat surface ${ }^{36,39,40}$. The atomic arrangements of atomic-flat and -stepped surface are depicted in Supplementary Fig. 6. Then, CVD was performed to synthesize BLGs on the $\mathrm{Cu}$ surface. After synthesizing BLGs on the $\mathrm{Cu}(311) /(110)$, the grown BLGs were characterized by SEM. Figure $2 b$ shows an SEM image of the resulting BLGs. It shows that all BLGs have twisted stacking order with various stacking angles. The stacking angle of each BLG is displayed with yellow dashed lines. To obtain the statistical information for the distribution of the stacking angle of BLG, we measured the stacking angles for $250 \mathrm{BLGs}$. The statistic indicates that $3^{\circ}-4^{\circ}-$, and $5^{\circ}$-twisted BLGs are the major products, and $46 \%$ of BLGs have stacking angles below $5^{\circ}$ (Fig. 2c). To confirm the 

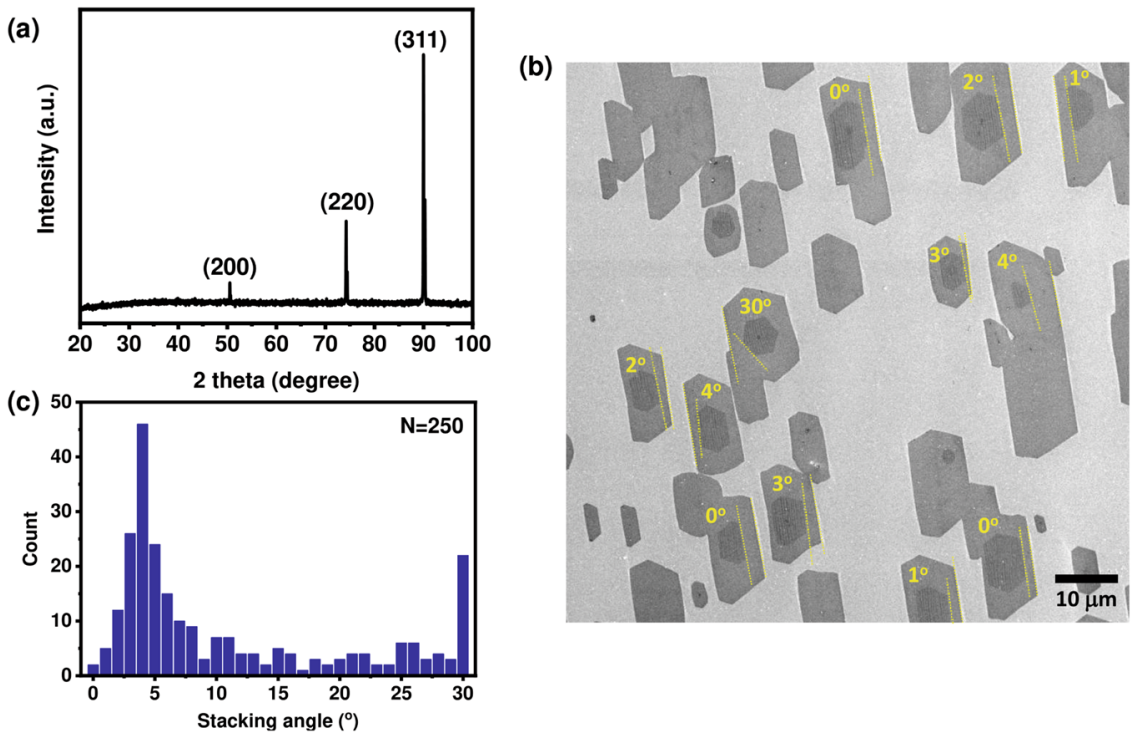

Fig. 2 Characterization of $\mathbf{C u}(\mathbf{3 1 1}) /(\mathbf{1 1 0})$ and grown BLG. a XRD pattern of $\mathrm{Cu}(311) /(110)$. b SEM image of BLG/Cu (311)/(110). The stacking angles of each BLG are indicated by yellow dashed lines. c Stacking angle distribution of BLGs grown on Cu (311)/(110). The most preferential stacking angle on $\mathrm{Cu}(311) /(110)$ is below $5^{\circ}$.

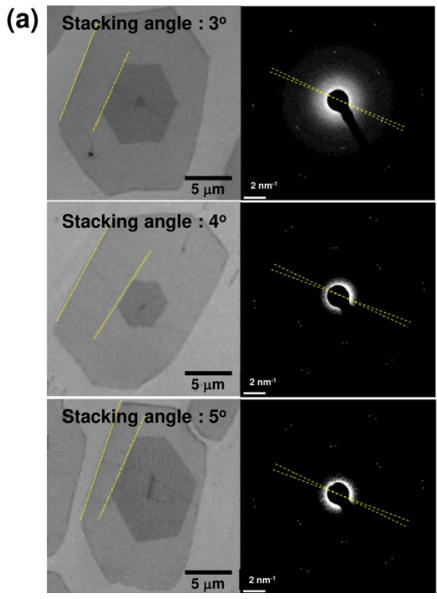

(b)

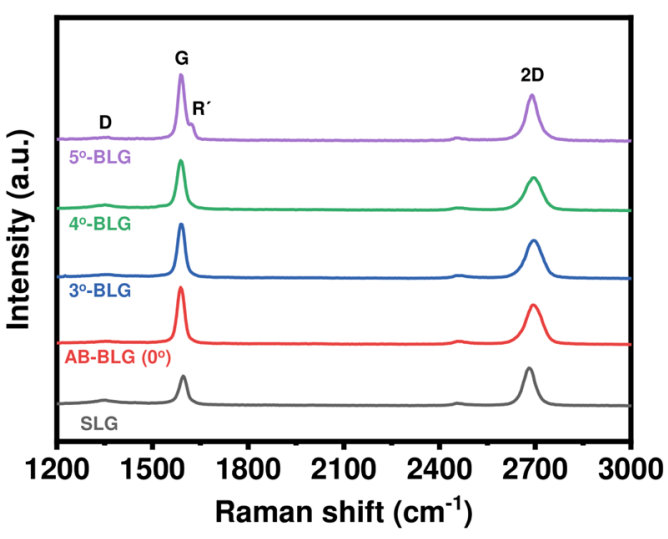

Fig. 3 Structural and spectroscopic characterization of small-angle twisted BLG. a SEM images (left) and SAED patterns (right) of BLGs with $3^{\circ}-, 4^{\circ}-$, and $5^{\circ}$-twisted stacking angle. The stacking angles of BLG are indicated by yellow dashed lines. b Raman spectra of SLG (black), AB-

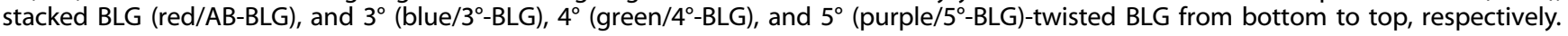

reproducibility of such a preference of stacking angle, we further attempted BLG growth on freshly prepared $5 \mathrm{Cu}(311) /(110)$ substrates, and the average percentage of BLG with below $5^{\circ}$ was 45.8\% (Supplementary Fig. 7).

Characterization of small-angle twisted BLG

The BLGs having preferred small stacking angles were characterized by SEM, SAED, and Raman spectroscopy. The SEM images of BLGs that have most preferential small stacking angles $\left(3-5^{\circ}\right)$ and their corresponding SAED patterns are shown in the left and right column of Fig. 3a, respectively. The stacking angles of BLG are measured from the rotated angle between two hexagonal diffraction patterns, and confirmed that the corresponding SAED patterns are rotated at $-3^{\circ}, 4^{\circ}$, and $5^{\circ}$, which is well-matched with the results observed from each SEM image of BLGs. In addition, we also obtained the SAED patterns of $1^{\circ}$ - and $2^{\circ}$-twisted BLG (Supplementary Fig. 8). The BLGs are further characterized by Raman spectroscopy with $532 \mathrm{~nm}$ excitation wavelength. The Raman spectra of BLG exhibit clearly different features from the SLG according to stacking angles, such as intensity of the G-band, intensity ratio of $2 \mathrm{D}$ - and G-band $\left(I_{2 \mathrm{D}} / \mathrm{I}_{\mathrm{G}}\right)$, and the full width at half maximum (FWHM) of 2D-band ${ }^{41-43}$. As shown in Fig. $3 \mathrm{~b}$, all the BLGs show higher intensity of the G-band than SLG. Since the Gband is induced by in-plane vibration of $\mathrm{sp}^{2}$ carbon atoms, the intensity of the G-band becomes stronger as the number of graphene layers increases ${ }^{44,45}$. In addition, the $5^{\circ}$-twisted BLG shows a new band at around $1612 \mathrm{~cm}^{-1}$ where the $\mathrm{R}^{\prime}$-band appears. It is known that the $\mathrm{R}^{\prime}$ mode is attributed to the superlattice modulation that activates phonons in the interior of Brillouin zone, and the $\mathrm{R}^{\prime}$-band is also known to appear when the stacking angle of BLG is between $5^{\circ}$ and $9^{\circ 46-48}$. Supplementary Table 1 shows the value of FWHM for the 2D-band and $I_{2 \mathrm{D}} / I_{\mathrm{G}}$ in SLG and AB-stacked BLG and each small-angle BLG $\left(3^{\circ}, 4^{\circ}\right.$, and $\left.5^{\circ}\right)$. All BLGs show broader 2D-bands than SLG because of the splitting of phonon or electron bands due to the strong coupling interaction between the two layers ${ }^{41}$. Also, the 2D-band was red-shifted as the stacking angle increases. In addition, BLGs show lower $I_{2 \mathrm{D}} / I_{\mathrm{G}}$ than SLG, and as the stacking angle increases from $0^{\circ}$ (AB stacking) to $5^{\circ}$, the FWHM values for the 2D-band become smaller. 
Growth mechanism of BLG on atomic-stepped Cu surface

While growth mechanism of BLG on Cu surface by CVD is still under debate, there are two growth mechanisms widely accepted. One is an on-top growth mechanism which describes that the second layer of BLG grows on top of the first layer ${ }^{19}$. The other one is the underlayer growth mechanism which describes that the second layer of BLG grows under the first layer ${ }^{49-51}$. It is believed that the underlayer mechanism is more appropriate under the $\mathrm{H}_{2}$ rich CVD environment because of the following reason. Under the $\mathrm{H}_{2}$-rich environment, hydrogen atoms can be attached to the edge of the first layer of BLG, which will generate enough space for carbon atoms to diffuse between the first layer and the $\mathrm{Cu}$ surface. Then, it allows the second layer growth of BLG under the first layer ${ }^{52}$.

To reveal the growth mechanism and stacking angle determination process of BLG on (311) and (110) surfaces, we measured XRD and SEM images according to the growth time. Also, the time-temperature graph for BLG growth process and detailed information about the changes of Cu surface and BLG growth are described in supporting information with Supplementary Fig. 9. Supplementary Fig. 10a shows the XRD of the $\mathrm{Cu}(311) /(110)$ surface at various growth times. It should be noted that (311)/ (110) plane is maintained for the first $10 \mathrm{~min}$, but when the growth time is over $20 \mathrm{~min}$, Cu becomes dominated with (111) at growth temperature of $1050^{\circ} \mathrm{C}$. The SEM images measured according to the BLG growth time show that the first layer of BLG is nucleated and grows at $3 \mathrm{~min}$ (inset of Supplementary Fig. 10b), and the second layer of BLG appeared after 5 min (inset of Supplementary Fig. 10b), of which Cu surface is still $(311) /(110)$ with unaligned BLG islands (alignment of BLGs is displayed with yellow dashed line). After being changed to (111), the resulting BLGs are found to be aligned in the same direction, which is displayed with red dashed lines. From these results, it is confirmed that the stacking angle of BLG is decided by $\mathrm{Cu}(311) /(110)$ surface, while the overall alignment occurs while aligning with (111) direction. It is noteworthy that the small stacking angle of BLG is still maintained after the alignment because the strong interlayer interaction between the first and the second layer of BLG is already formed in BLG growth step when the Cu still has (311) and (110) crystal planes.

DFT calculation of BLG on atomic-flat and -stepped Cu surface The preferred growth of BLG with specific stacking angles on $\mathrm{Cu}$ (111) and $\mathrm{Cu}(311) /(110)$ is closely related to the different preferential formations of graphene crystal lattice on each surface. To evaluate the preferred stacking angle on atomic-flat and -stepped surfaces, we calculated the stabilization energy of BLG for AB-stacking, small-angle twisted stacking (below $5^{\circ}$ ), and $30^{\circ}$ twisted stacking on both surfaces. Detailed calculation procedure is described in Supplementary information. For clarification, we

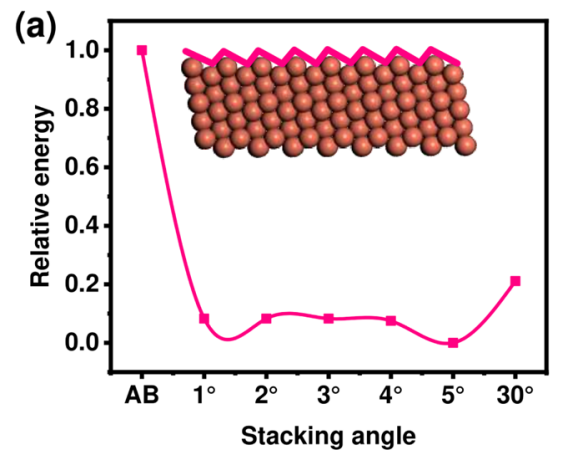

divided the formation of BLG on both atomic-flat and -stepped surfaces into two steps. The first step is the formation of SLG on the $\mathrm{Cu}$ surface, of which location is determined by carbon adsorption energies. The second step is the formation of the second layer under the SLG, which is mainly affected by the interactions with $\mathrm{Cu}$ surface and SLG. Using density-functional theory (DFT) calculations, we confirmed the energetically preferred stacking angles of BLG on both $\mathrm{Cu}$ surfaces. When we calculate the preferred positions for SLG on $\mathrm{Cu}$ (311) and (110) surfaces using a graphene flake model composed of 54 carbon atoms, it is found that SLG becomes most stable when carbons occupy all the possible adsorption sites of $\mathrm{Cu}$ surfaces (seven sites noted as $T, T^{\prime}, B, B^{\prime}, H_{h}, H_{f}$, and $H_{4}$ for (311) and five sites noted as $T, B, B^{\prime}, H_{4}$, and $H_{h}$ for (110)) as shown in Supplementary Fig. $11 \mathrm{~b}$, $C$, respectively ${ }^{53-55}$. We also confirmed that the most stable SLG is formed when the carbon atoms are at the top $(T)$ and hollow $\left(H_{h}\right)$ sites on Cu (111) (Supplementary Fig. 11a). Based on the SLG results, we calculated the stabilization energy for BLG at various stacking angles on each $\mathrm{Cu}$ surface. Figure $4 \mathrm{a}$, b shows relative energies depending on the stacking angle on each surface. On $\mathrm{Cu}$ (111), BLG with $A B$-stacking and $30^{\circ}$-twisted stacking structure was more stable than the small-angle twisted structure (below $5^{\circ}$ ), which agrees well with previous studies (Supplementary Fig. 11d). On the other hand, the relative stabilization energy of small-angle twisted BLG is much lower than AB-stacking on both (311) and (110) surfaces (Fig. 4a, b). It should be noted that $30^{\circ}$-twisted stacking energy of BLG is slightly higher than the small-angle twisted form. From these results, it can be explained that the formation of small-angle twisted BLGs is energetically favored on the atomic-stepped Cu surface.

In summary, we found experimental evidence that the structure of the $\mathrm{Cu}$ surface determines the stacking angle of BLG. The surface that has atomic-stepped structure, such as $\mathrm{Cu}(311) /(110)$ surface, results in small-angle twisted stacking of BLGs with preferential stacking angles of below $5^{\circ}$, while the surface that has an atomic-flat structure, like (111) surface of $\mathrm{Cu}$ preferentially produces $\mathrm{AB}$-stacked $\mathrm{BLG}$ and $30^{\circ}$-twisted BLGs. The stacking angle of BLG and the relationship between the $\mathrm{Cu}$ surface and the BLG were analyzed by SEM, SAED, XRD, and Raman spectroscopy. Through DFT energy calculation of BLG on each Cu surface, we revealed that the small-angle twisted BLGs are energetically more favored on atomic-stepped $\mathrm{Cu}$ surface. Our findings show that fine-tuning of the stacking angle of BLG could be possible with further fine-tuning of $\mathrm{Cu}$ catalyst surface.

\section{METHODS}

Synthesis of BLG by CVD process

A piece of $0.02 \mathrm{~mm}$ thick Cu foil (Nilaco, model \# Cu-113213) is soaked in acetic acid for $1 \mathrm{~min}$ to remove surface oxide and impurities. Then the $\mathrm{Cu}$

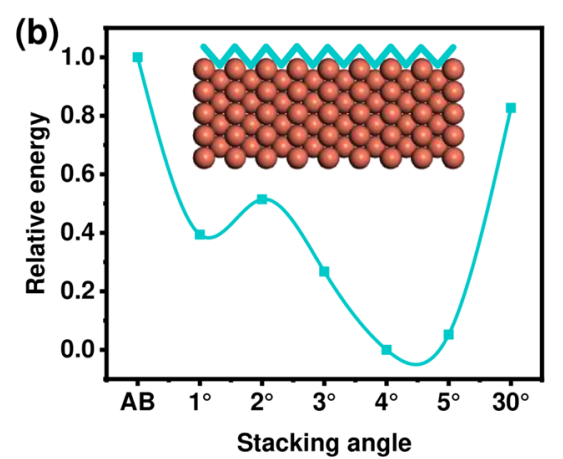

Fig. 4 Stacking angle-relative energy graph of BLG depending on stacking angle. a BLG on Cu (311) and $\mathbf{b}$ BLG on Cu (110). Insets are the illustration for the cross-section of each crystal plane. The atomic-stepped surface morphology of (311) and (110) is displayed by pink and cyan lines, respectively. 
foil is dried with $\mathrm{N}_{2}$ gas. The $\mathrm{Cu}$ foil $(2 \mathrm{~cm} \times 3.5 \mathrm{~cm})$ is loaded in the middle of a quartz tube ( $1 \mathrm{in}$. diametre) that is placed at the center of a tube-type heating furnace (Lindberg/Blue M, model \# TF55030C-1), and the system is evacuated under vacuum for $10 \mathrm{~min}$. After the evacuation, Ar gas (purity: 99.999\%, Linde) and $\mathrm{H}_{2}$ gas (purity: $99.9999 \%$, Daesung) are supplied at 300 and $100 \mathrm{sccm}$ of flow rate, respectively, into the quartz tube, and the temperature of the furnace is raised up to $1000^{\circ} \mathrm{C}$ to prepare $\mathrm{Cu}(111)$ and to $800{ }^{\circ} \mathrm{C}$ for $\mathrm{Cu}(311) /(110)$, then kept with a flow of $300 \mathrm{sccm}$ Ar and $100 \mathrm{sccm} \mathrm{H} \mathrm{H}_{2}$ for $2 \mathrm{~h}$ under 5 Torr for annealing. After the annealing process, the flow rates for $\mathrm{Ar}$ and $\mathrm{H}_{2}$ gas are changed to $100 \mathrm{sccm}$ and $250 \mathrm{sccm}$, respectively, and the furnace temperature is set to $1050{ }^{\circ} \mathrm{C}$ for the growth of BLG. In addition, $20 \mathrm{sccm}$ of dilute $\mathrm{CH}_{4}$ gas (1\% mol in $\mathrm{H}_{2}$, RIGAS) is supplied as a carbon source when the furnace temperature reaches $1050^{\circ} \mathrm{C}$ for $30 \mathrm{~min}$ under 20 Torr. After the growth, the furnace is rapidly cooled to room temperature by opening the top lid of the tube furnace still with a flow of $100 \mathrm{sccm} \mathrm{Ar}$ and $250 \mathrm{sccm} \mathrm{H}$.

\section{Bilayer graphene transfer}

BLGs are transferred onto a $300 \mathrm{~nm} \mathrm{SiO} / \mathrm{Si}$ substrate by a wet transfer method for characterization. To transfer the prepared BLGs, one side of a Cu/BLG surface is spin-coated with PMMA (Microchem, 950 PMMA A5), and cured at $180^{\circ} \mathrm{C}$ for $1 \mathrm{~min}$. The other side of the $\mathrm{Cu} / \mathrm{BLG}$ is treated by $\mathrm{O}_{2}$ plasma for $5 \mathrm{~min}$ to remove graphene. The oxygen flow rate is $10 \mathrm{sccm}$ with power set at $200 \mathrm{~W}$. The sample is floated on a $0.5 \mathrm{M}$ ammonium persulfate (Sigma-Aldrich, >98.0\%) solution to etch the $\mathrm{Cu}$ for $6 \mathrm{~h}$. After etching the $\mathrm{Cu}$, the freestanding PMMA/BLG sample is washed with $\mathrm{DI}$ water several times and transferred onto a $\mathrm{SiO}_{2}$ substrate. After transfer, the sample is annealed at $200^{\circ} \mathrm{C}$ in air for $2 \mathrm{~h}$ to increase the adhesion between the $\mathrm{SiO}_{2}$ substrate and the BLG. The sample is then soaked in acetone for $30 \mathrm{~min}$ to remove PMMA. To get rid of PMMA residue, the BLG/ $\mathrm{SiO}_{2}$ sample is annealed at $250^{\circ} \mathrm{C}$ for $2 \mathrm{~h}$ in the flow of $1000 \mathrm{sccm}$ Ar and $500 \mathrm{sccm} \mathrm{H}$.

\section{Characterizations}

The crystal plane of Cu catalyst is analyzed using an X-ray diffractometer (D/MAX-2500/PC). The scan range is $20^{\circ}-100^{\circ}$ with a scan rate of $0.06^{\circ} / \mathrm{s}$. The crystal plane distribution for the whole area is analyzed using EBSD (Edax, Hikari XP EBSD Camera). Raman spectra and mapping images are obtained using a WITECH Alpha 300R Raman spectroscope equipped with a Nd:YAG laser. The excitation wavelength is $532 \mathrm{~nm}$. The FWHM values of the 2D-band are extracted from $100 \%$ Lorentzian profile of each spectrum. The SAED patterns are obtained using a HR-FE-TEM-(2200FS with Cscorrected TEM). The intensity profile of each SAED pattern is analyzed using Gatan Digital Microscope program.

\section{Computational details}

DFT calculations were performed to calculate the stabilization energy for the stacking angle of BLG on atomic-flat and atomic-stepped $\mathrm{Cu}$ surfaces. We used $\mathrm{DMol}^{3}$ module in Material Studio program and the double numerical plus polarization (DNP) was used as a basis set. Before BLG calculation on Cu surface, we optimized the geometry of SLG on each Cu surface. After finding the preferential position of SLG, we located the second layer of BLG, which consists of 54 carbon atoms under the stabilized SLG. It is noted that the interlayer distance between the first and the second layer was $0.34 \mathrm{~nm}$ that is the van der Waals distance. The stabilization energies of BLG were obtained according to the stacking angle.

\section{DATA AVAILABILITY}

All principal data with detailed experimental procedure and characterization of this work are included in this paper and its supporting information.

Received: 31 March 2020; Accepted: 15 September 2020; Published online: 13 October 2020

\section{REFERENCES}

1. McCann, E. \& Koshino, M. The electronic properties of bilayer graphene. Rep. Prog. Phys. 76, 056503 (2013).
2. Ohta, T., Bostwick, A., Seyller, T., Horn, K. \& Rotenberg, E. Controlling the electronic structure of bilayer graphene. Science 313, 951-954 (2006).

3. Yan, H. Bilayer graphene: physics and application outlook in photonics. Nanophotonics 4, 115-127 (2015).

4. Zhang, Y. et al. Direct observation of a widely tunable bandgap in bilayer graphene. Nature 459, 820-823 (2009).

5. Sato, Y., Takai, K. \& Enoki, T. Electrically controlled adsorption of oxygen in bilayer graphene devices. Nano Lett. 11, 3468-3475 (2011).

6. Choi, Y. et al. Electronic correlations in twisted bilayer graphene near the magic angle. Nat. Phys. 15, 1174-1180 (2019).

7. Codecido, E. et al. Correlated insulating and superconducting states in twisted bilayer graphene below the magic angle. Sci. Adv. 5, eaaw9770 (2019).

8. Culchac, F. J., Del Grande, R. R., Capaz, R. B., Chico, L. \& Morell, E. S. Flat bands and gaps in twisted double bilayer graphene. Nanoscale 12, 5014 (2020).

9. Cao, Y. et al. Unconventional superconductivity in magic-angle graphene superlattices. Nature 556, 43-50 (2018).

10. Yankowitz, M. et al. Tuning superconductivity in twisted bilayer graphene. Science 363, 1059-1064 (2019).

11. Patel, H., Huang, L., Kim, C.-J., Park, J. \& Graham, M. W. Stacking angle-tunable photoluminescence from interlayer exciton states in twisted bilayer graphene. Nat. Commun. 10, 1445 (2019).

12. Brihuega, I. et al. Unraveling the intrinsic and robust nature of van Hove singularities in twisted bilayer graphene by scanning tunneling microscopy and theoretical analysis. Phys. Rev. Lett. 109, 196802 (2012).

13. Ribeiro, H. B. et al. Origin of van Hove singularities in twisted bilayer graphene. Carbon 90, 138-145 (2015).

14. Zhang, Y., Zhang, L. \& Zhou, C. Review of chemical vapor deposition of graphene and related applications. Acc. Chem. Res. 46, 2329-2339 (2013).

15. Muñoz, R. \& Gómez-Aleixandre, C. Review of CVD synthesis of graphene. Chem. Vap. Depos. 19, 297-322 (2013).

16. Li, X., Colombo, L. \& Ruoff, R. S. Synthesis of graphene films on copper foils by chemical vapor deposition. Adv. Mater. 28, 6247-6252 (2016).

17. Nguyen, V. L. et al. Wafer-scale single-crystalline AB-stacked bilayer graphene. Adv. Mater. 28, 8177-8183 (2016).

18. Lee, J.-K. et al. The nature of metastable $A A^{\prime}$ graphite: low dimensional nano- and single-crystalline forms. Sci. Rep. 6, 39624 (2016).

19. Fang, W., Hsu, A. L., Song, Y. \& Kong, J. A review of large-area bilayer graphene synthesis by chemical vapor deposition. Nanoscale 7, 20335-20351 (2015).

20. Yan, Z. et al. Large hexagonal bi- and trilayer graphene single crystals with varied interlayer rotations. Angew. Chem. 53, 1565-1569 (2014).

21. Deng, B. et al. Interlayer decoupling in $30^{\circ}$ twisted bilayer graphene quasicrystal. ACS Nano 14, 1656-1664 (2020).

22. Halle, J., Mehler, A., Néel, N. \& Kröger, J. Preparation of graphene bilayers on platinum by sequential chemical vapour deposition. Phys. Chem. Chem. Phys. 21 3140-3144 (2019).

23. Qian, Y. \& Kang, D. J. Large-area high-quality AB-stacked bilayer graphene on hBN/Pt foil by chemical vapor deposition. ACS Appl. Mater. Interfaces 10, 29069-29075 (2018).

24. Reina, A. et al. Growth of large-area single- and bi-layer graphene by controlled carbon precipitation on polycrystalline Ni surfaces. Nano Res. 2, 509-516 (2009).

25. Song, Y. et al. Epitaxial nucleation of CVD bilayer graphene on copper. Nanoscale 8, 20001-20007 (2016)

26. Zhang, W., Wu, P., Li, Z. \& Yang, J. First-principles thermodynamics of graphene growth on Cu surfaces. J. Phys. Chem. C 115, 17782-17787 (2011).

27. Losurdo, M., Giangregorio, M. M., Capezzuto, P. \& Bruno, G. Graphene CVD growth on copper and nickel: role of hydrogen in kinetics and structure. Phys. Chem. Chem. Phys. 13, 20836 (2011).

28. Li, X., Cai, W., Colombo, L. \& Ruoff, R. S. Evolution of graphene growth on Ni and Cu by carbon isotope labeling. Nano Lett. 9, 4268-4272 (2009).

29. Nie, S. et al. Growth from below: Bilayer graphene on copper by chemical vapor deposition. New J. Phys. 14, 093028 (2012).

30. Liu, H. \& Liu, Y. Controlled chemical synthesis in CVD graphene. Phys. Sci. Rev. 2, 1-28 (2017).

31. Yang, N., Choi, K., Robertson, J. \& Park, H. G. Layer-selective synthesis of bilayer graphene via chemical vapor deposition. 2D Mater. 4, 035023 (2017).

32. Nie, S. et al. Growth from below: graphene bilayers on $\operatorname{Ir}(111)$. ACS Nano $\mathbf{5}$, 2298-2306 (2011).

33. Yan, K., Peng, H., Zhou, Y., Li, H. \& Liu, Z. Formation of bilayer bernal graphene: layer-by-layer epitaxy via chemical vapor deposition. Nano Lett. 11, 1106-1110 (2011).

34. Cousins, C. S. G. Elasticity of carbon allotropes. IV. Rhombohedral graphite: elasticity, zone-center optic modes, and phase transformation using transferred Keating parameters. Phys. Rev. B 67, 024110 (2003). 
35. Ishikawa, A., Doi, T. \& Nakai, H. Catalytic performance of Ru, Os, and Rh nanoparticles for ammonia synthesis: a density functional theory analysis. J. Catal. 357, 213-222 (2018).

36. Leroy, F., Müller, P., Métois, J. J. \& Pierre-Louis, O. Vicinal silicon surfaces: from step density wave to faceting. Phys. Rev. B Condens. Matter 76, 1-15 (2007).

37. Ju Wook, L., Jeongyong, L., Sang-Gi, K., Kee-Soo, K. \& Jin-Gun, K. Structural modification of a trench by hydrogen annealing. J. Korean Phys. Soc. 37, 1034 (2000).

38. Otte, H. M. \& Crocker, A. G. Crystallographic formulae for hexagonal lattices. Phys. Status Solidi 9, 441-450 (1965).

39. Walen, $\mathrm{H}$. et al. Reconstruction of steps on the $\mathrm{Cu}(111)$ surface induced by sulfur. J. Chem. Phys. 142, 194711 (2015).

40. Gu, C. et al. Growth of quasi-free-standing single-layer blue phosphorus on tellurium monolayer functionalized Au(111). ACS Nano 11, 4943-4949 (2017).

41. Popov, V. N. 2D Raman band of single-layer and bilayer graphene. J. Phys. Conf. Ser. 682, 012013 (2016).

42. Ferrari, A. C. et al. Raman spectrum of graphene and graphene layers. Phys. Rev. Lett. 97, 187401 (2006).

43. Wu, J.-B. et al. Resonant Raman spectroscopy of twisted multilayer graphene. Nat. Commun. 5, 5309 (2014).

44. Reich, S. \& Thomsen, C. Raman spectroscopy of graphite. Philos. Trans. R. Soc. A Math. Phys. Eng. Sci. 362, 2271-2288 (2004).

45. Ni, Z., Wang, Y., Yu, T. \& Shen, Z. Raman spectroscopy and imaging of graphene. Nano Res. 1, 273-291 (2008).

46. Niilisk, A. et al. Raman modes in transferred bilayer CVD graphene. Open Phys. 13, 34-40 (2015).

47. Schmucker, S. W. et al. Raman signature of defected twisted bilayer graphene. Carbon 93, 250-257 (2015).

48. Kim, K. et al. Raman spectroscopy study of rotated double-layer graphene: misorientation-angle dependence of electronic structure. Phys. Rev. Lett. 108, 246103 (2012).

49. $\mathrm{Wu}, \mathrm{B}$. et al. Equiangular hexagon-shape-controlled synthesis of graphene on copper surface. Adv. Mater. 23, 3522-3525 (2011).

50. Wu, J. et al. Synchronous growth of high-quality bilayer bernal graphene: From hexagonal single-crystal domains to wafer-scale homogeneous films. Adv. Funct. Mater. 27, 1-8 (2017).

51. Yi, D., Jeon, S. \& Hong, S. W. Selectively patterned regrowth of bilayer graphene for self-integrated electronics by sequential chemical vapor deposition. ACS Appl. Mater. Interfaces 10, 40014-40023 (2018).

52. Zhang, X., Wang, L., Xin, J., Yakobson, B. I. \& Ding, F. Role of hydrogen in graphene chemical vapor deposition growth on a copper surface. J. Am. Chem. Soc. 136, 3040-3047 (2014).

53. Spencer, M. J. S. \& Nyberg, G. L. DFT modelling of hydrogen on $\mathrm{Cu}(110)$ - and (111)-type clusters. Mol. Simul. 28, 807-825 (2002).

54. Tian, F. H. \& Wang, Z. X. Adsorption of an $\mathrm{O}$ atom on the $\mathrm{Cu}$ (311) step defective surface. J. Phys. Chem. B 108, 1392-1395 (2004).

55. Liao, K., Fiorin, V., Gunn, D. S. D., Jenkins, S. J. \& King, D. A. Single-crystal adsorption calorimetry and density functional theory of CO chemisorption on fcc Co\{110\}. Phys. Chem. Chem. Phys. 15, 4059 (2013).

\section{ACKNOWLEDGEMENTS}

Observation of morphologies and electron diffraction patterns of BLG using scanning electron microscope (SEM) and high resolution transmission electron microscope (HR-TEM) were carried out at the National Institute for Nanomaterials Technology (NINT) in Pohang, Korea. This research was supported by Samsung Electronics and Veteran researcher grant (No. 2019R1A2C2004259) managed by National Research Foundation of Korea (NRF).

\section{AUTHOR CONTRIBUTIONS}

H.C.C., H.C., and Y.P. conceived and designed the experiments. Y.P. and H.C. performed experiments, and H.C.C., H.C., and Y.P. co-wrote the paper. S.K. measured and analyzed the TEM-SAED patterns of BLG and CU. T.A. and T.H.K. conducted the STM measurements.

\section{COMPETING INTERESTS}

The authors declare no competing interests.

\section{ADDITIONAL INFORMATION}

Supplementary information is available for this paper at https://doi.org/10.1038/ s41699-020-00169-x.

Correspondence and requests for materials should be addressed to H.C.C.

Reprints and permission information is available at http://www.nature.com/ reprints

Publisher's note Springer Nature remains neutral with regard to jurisdictional claims in published maps and institutional affiliations.

(i) Open Access This article is licensed under a Creative Commons Attribution 4.0 International License, which permits use, sharing, adaptation, distribution and reproduction in any medium or format, as long as you give appropriate credit to the original author(s) and the source, provide a link to the Creative Commons license, and indicate if changes were made. The images or other third party material in this article are included in the article's Creative Commons license, unless indicated otherwise in a credit line to the material. If material is not included in the article's Creative Commons license and your intended use is not permitted by statutory regulation or exceeds the permitted use, you will need to obtain permission directly from the copyright holder. To view a copy of this license, visit http://creativecommons. org/licenses/by/4.0/.

(c) The Author(s) 2020 